\title{
Small Loop Antenna System Design for Radio Direction Finding
}

\author{
Tain-Sou Tsay \\ Department of Aeronautical Engineering, \\ National Formosa University, \\ No.64, Wen-Hua Road, Huwei, Yunlin, TAIWAN
}

\begin{abstract}
In this literature, a low-cost small loop antenna is developed for radio direction finding. It consists of two coupled rectangular counter-wiring loop antennas. A signal-processing circuit is developed also for demodulated outputs. A single rectangular loop antenna is discussed first for illustrating the receiving characteristics and then the proposed two coupled rectangular counter-wiring loop antennas are designed for radio direction finding. Measurements give a large linear detecting range. It is ready for Omni-directional application using another two coupled loop antennas and can be used as a tracking control device.
\end{abstract}

Key-Words: - small loop antenna, radio direction finding, tracking control device, signal processing circuits.

Received: May 25, 2021. Revised: September 27, 2021. Accepted: November 25, 2021. Published: December 16, 2021.

\section{Introduction}

Loop antennas (such as circular, square, triangular, rectangular, and rhombic loops) have been used since the early days of radio, and are in common use at many frequencies below about $1 \mathrm{GHz}$. They have become widespread for applications in LF and HF band, particularly for mobile communications on vehicles using nearvertical-incidence skywave propagation mode [1-3].

The small loop antenna is the possible solution for LF and HF bands [4, 5]. Small loop antennas are less than $0.1 \lambda$ and respond to the magnetic field of the electromagnetic wave. The disadvantages of the small loop antenna are (1) poor efficiency, (2) lower frequency application. The advantages of the small loop antenna are (1) light in weight, (2) simple and compact in structure, (3) economic, (4) portable application.

The rectangular multi-Loop antenna is usually used for implementing it easily [6]. The maximal and minimal outputs of the single loop antenna are well discussed. It cannot identify the incidence angle of the electromagnetic wave. The proposed loop antenna consists of two identical loop antennas [7, 8]. The dimension of loop antennas and their spacing are optimized. Then, it becomes a directional antenna for radio radiation detection with the proposed signal processing circuit.

In this paper, the rectangular single-loop antenna is implemented first for designing the signalprocessing circuit and analyzing the characteristics of the loop antenna. Then the two coupled-loop antennas and the signal-processing circuit with demodulation are proposed for radio direction finding. Measurements give a large linear detecting range. It is ready for Omni-directional application using another two coupled loop antennas and can be used as a tracking control device.

\section{Loop Antenna Design}

\subsection{Single-Loop Antenna}

Fig. 1 shows the configuration of a single-loop antenna. The \#26 wires are used. The dimension of the loop antenna is $\mathrm{H}=0.93 \mathrm{~cm}, \mathrm{~W}=2.9 \mathrm{~cm}$; and $\mathrm{L}=9$. $5 \mathrm{~cm}$. The rule of thumb of them is induced as $\mathrm{L}>\mathrm{W}$ $>\mathrm{H}$. The longer $\mathrm{L}$ is, the better the measured resolution will be. The inductance of the loop is $121 \mathrm{uH}$.

Fig.2 is the signal pre-processing circuit. A varactor $(10 \sim 100 \mathrm{pf})$ is used for fine frequency tuning to $1 \mathrm{MHz}$; the first stage using a JFET (MPF-102) is selected for high input impedance for receiving the input signal; the second stage using an NPN Transistor (BC547B) is designed for signal amplification, and the third stage using an NPN Transistor (BC547B) is a buffer circuit for the output signal. Three coupling capacitors $(0.1 \mathrm{uF})$ are used for connecting three stages and filtering out the DC(direct current) signals. A variable resistor $(0 \sim 5 \mathrm{~K})$ in the second stage is used for adjusting the signal amplification gain; i.e., the output level of the 
alternative signal $V_{R}$. The operating point of each stage is designed for less signal-processing distortion compared with the sinusoidal input. The output $V_{R}$ is an $\mathrm{AC}($ Alternate Current) signal.

Table 1 gives peak values $V_{R}$ shown in Fig.2 for different values of heading angles heading to the signal source. Comparison is made for the ground shield is added or not. Fig. 3 shows the transfer curves using the datum of Table 1. It shows those symmetrical properties and cannot identify the right or left-hand side. This circuit is suitable for the use of human judgment joined. A two-loop antenna will be designed for left/right identification.

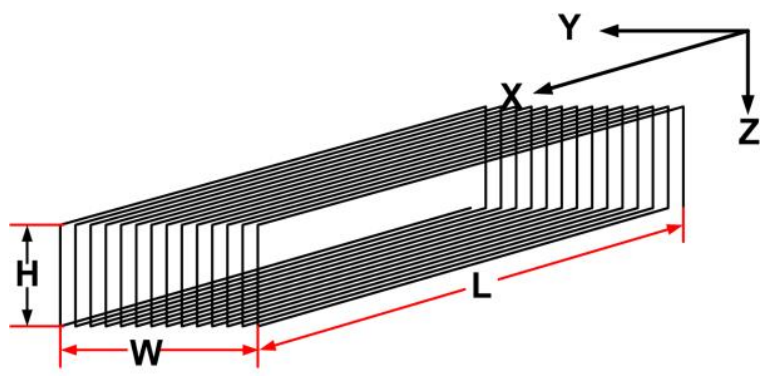

Fig.1. The Single-Loop Antenna.

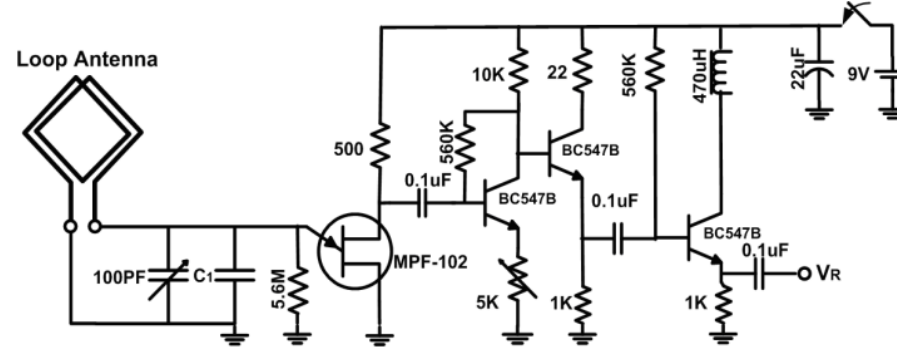

Fig.2. The Signal-Processing Circuit Design.

Table 1. Peak values of $V_{R}$ for Single-loop Antenna.

\begin{tabular}{|c|c|c|}
\hline \multirow{2}{*}{$\begin{array}{c}\text { Heading } \\
\text { Angle(degs.) }\end{array}$} & \multicolumn{2}{|c|}{$V_{R}$ (Volts) } \\
\cline { 2 - 3 } & $\begin{array}{c}\text { No Ground } \\
\text { Shield }\end{array}$ & $\begin{array}{c}\text { Has Ground } \\
\text { Shield }\end{array}$ \\
\hline 90.00 & $2.14 \sim 2.26$ & $1.78 \sim 1.81$ \\
\hline 60.00 & $2.46 \sim 2.54$ & $1.96 \sim 1.99$ \\
\hline 45.00 & $2.63 \sim 2.65$ & $2.04 \sim 2.07$ \\
\hline 30.00 & $2.70 \sim 2.74$ & $2.10 \sim 2.14$ \\
\hline 15.00 & $2.73 \sim 2.79$ & $2.18 \sim 2.19$ \\
\hline 0.00 & $2.78 \sim 2.80$ & $2.17 \sim 2.20$ \\
\hline-15.00 & $2.76 \sim 2.78$ & $2.17 \sim 2.18$ \\
\hline-30.00 & $2.68 \sim 2.70$ & $2.12 \sim 2.13$ \\
\hline-45.00 & $2.64 \sim 2.65$ & $2.08 \sim 2.09$ \\
\hline-60.00 & $2.55 \sim 2.53$ & $1.96 \sim 1.97$ \\
\hline-90.00 & $2.21 \sim 2.26$ & $1.75 \sim 1.78$ \\
\hline
\end{tabular}

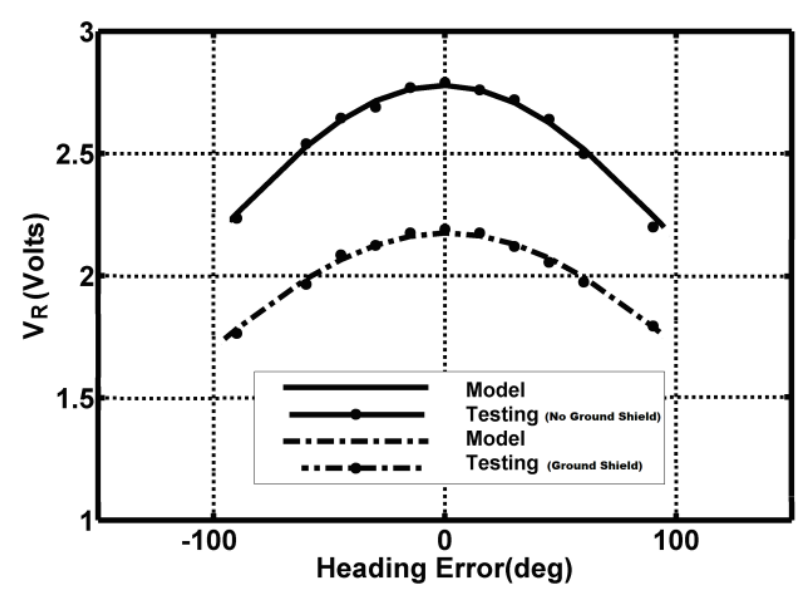

Fig.3. Transfer curve of single-loop antenna.

\subsection{The Proposed Loop Antenna-Double-loop Antenna}

The small loop antenna design for left/right side identification is shown in Fig.4. It consists of two rectangular wired loop-antennas. They are two counter-directional wiring loops. The \#26 wires are used. The dimension of each loop antenna is $\mathrm{H}=0$. $93 \mathrm{~cm}, \mathrm{~W}=2 \mathrm{~cm} ; \mathrm{L}=9.5 \mathrm{~cm}$, and the spacing $\mathrm{D}=1.70 \mathrm{~cm}$ between two loop antennas. The inductance of each loop is $82 \mathrm{uH}$.

Note that the maximal output of a single loop antenna is the incidence angle of the electromagnetic wave along the $\mathrm{X}$-axis, and the minimal output is the incidence angle of the electromagnetic wave along the Y-axis. However, the configuration shown in Fig.4 makes the sensing behaviors of two loops will be affected by each other. The maximal output of the left (or right) loop antenna is now not the incidence angle of the electromagnetic wave along the $\mathrm{X}$-axis for the sensing of electromagnetic wave of the left (or right) loop is affected by the right (left) antenna. It is called the coupling effect. The strength of the coupling effect is dependent on the spacing D of two loop antennas and will become to be a directional loop antenna for radio finding to identify the left/ right side. The above statements will be verified by real measurements.

Fig.5 shows signal processing circuits in which $V_{R}$ and $V_{L}$ are demodulated outputs in DC. Band-pass filters with inductance $470 \mathrm{uH}$ cascaded by variable capacitor $60 \mathrm{pF}$ are added for filtering out the receiving noise and demodulation circuits using a PNP transistor (2N3096), a Diode (1N914), a capacitor $(0.1 \mathrm{uF})$, and a resistor $(1 \mathrm{M})$ are added for filtering out the carrier frequency to find DC 
outputs. Another processing circuit using the linear antenna is added also to consider the ranging effect. The output of the circuit is $V_{\text {ref }}$. It is independent of the heading angle to the signal source while depending on the range between the antenna and the signal source. It is ready to consider the range effect The main attention in this work is to illustrate the linear property of the antenna that can be applied to another possible electronic product. The above statements will be verified by real measurements.

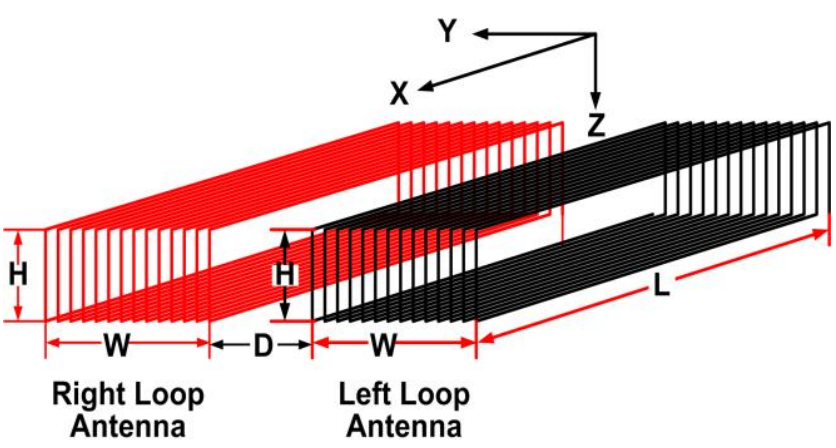

Fig.4. The geometry of the Proposed Loop Antenna.
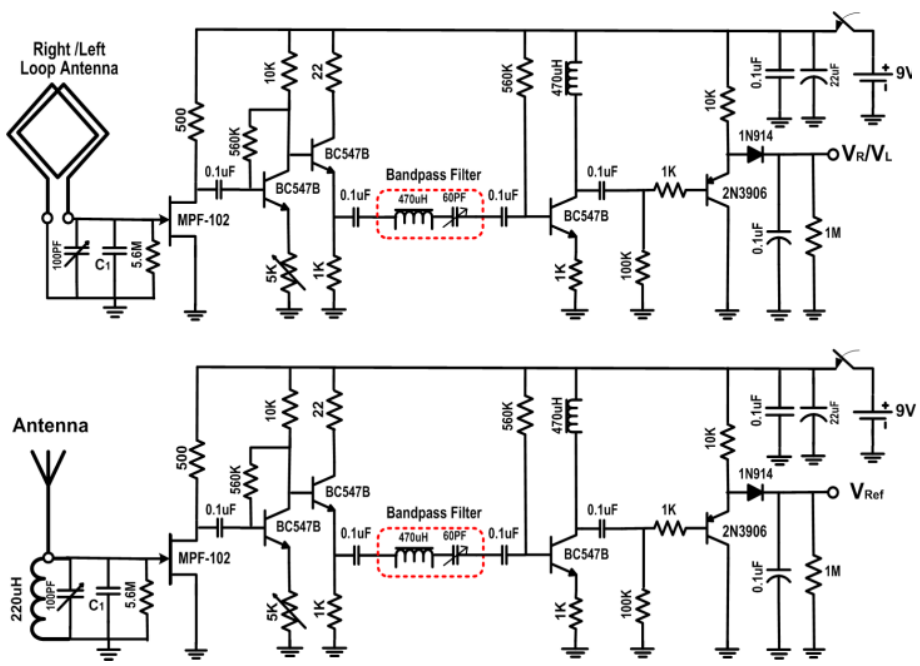

Fig.5. Signal processing circuits.

Figs.6-10 show testing results for heading angles are $0,+45,+90,-45$, and -90 degrees; respectively. The Tektronix $200 \mathrm{MHz}$ oscilloscope was used for measurements and data download. They show the processing circuit shown in Fig.5 can give stationary DC levels, and the low-pass filter using $0.1 \mathrm{uF}$ and $1 \mathrm{M} \Omega$ is suitable for the frequency of the signal source. Note that the output of the processing circuit shown in Fig. 2 is AC.

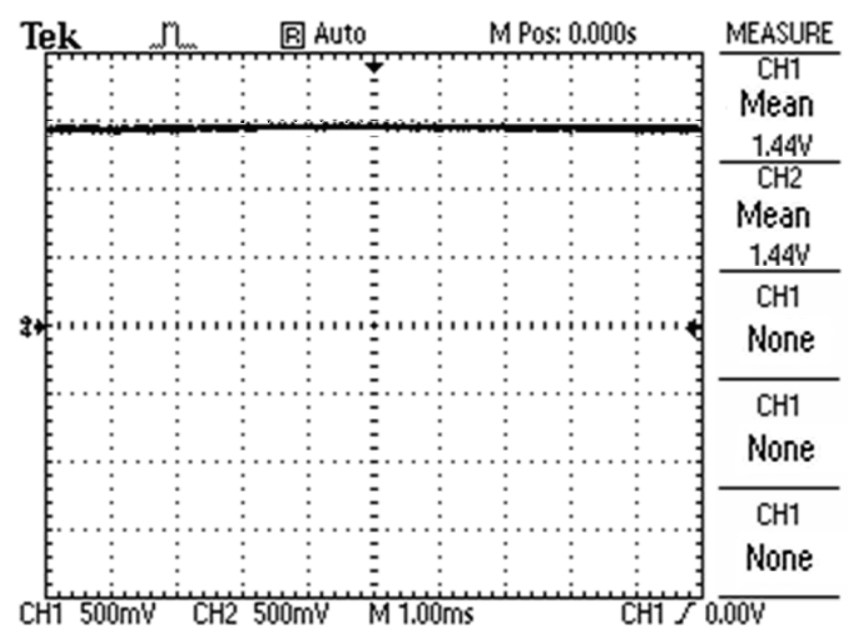

Fig.6. The testing result for the heading angle is 0degrees.

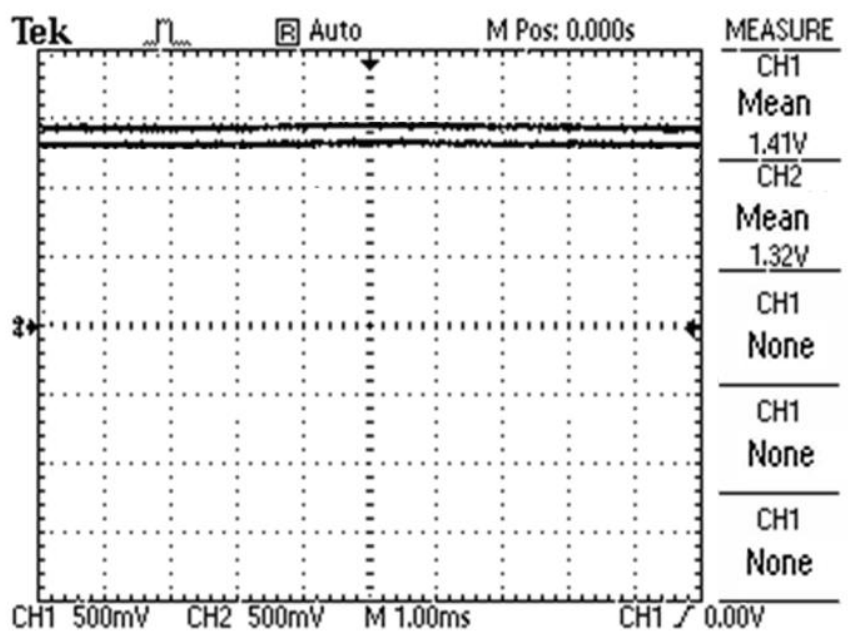

Fig.7. The testing result for the heading angle is +45degrees.

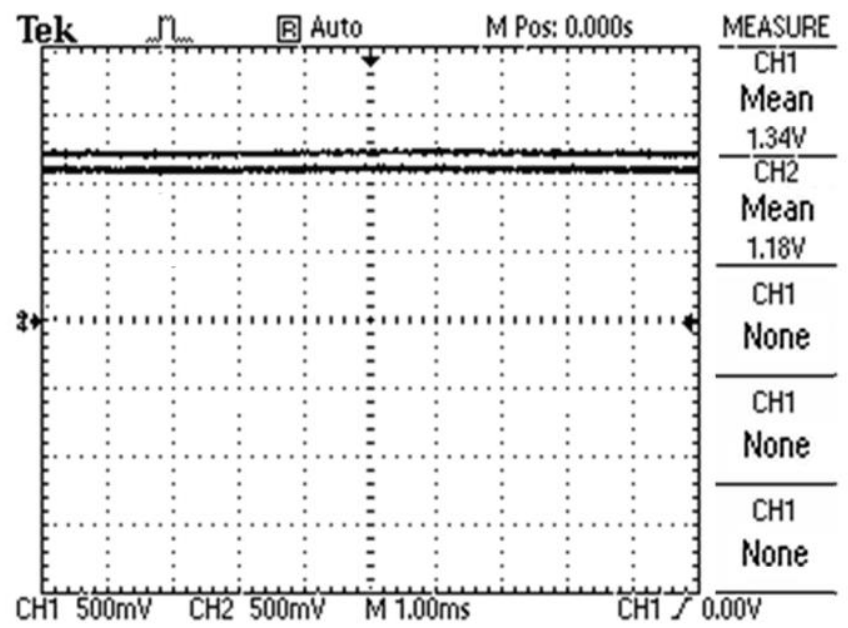

Fig.8. The testing result for the heading angle is +90degrees. 


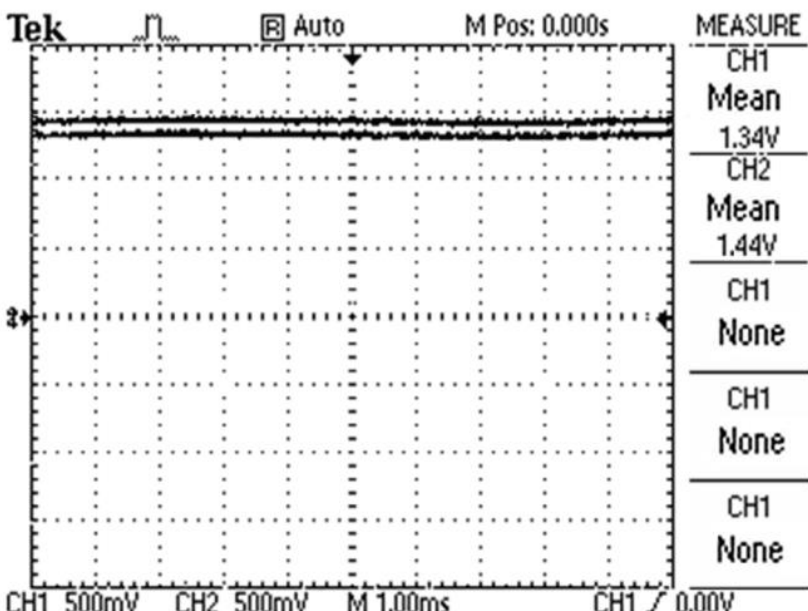

Fig.9. The testing result for the heading angle is -45degrees.

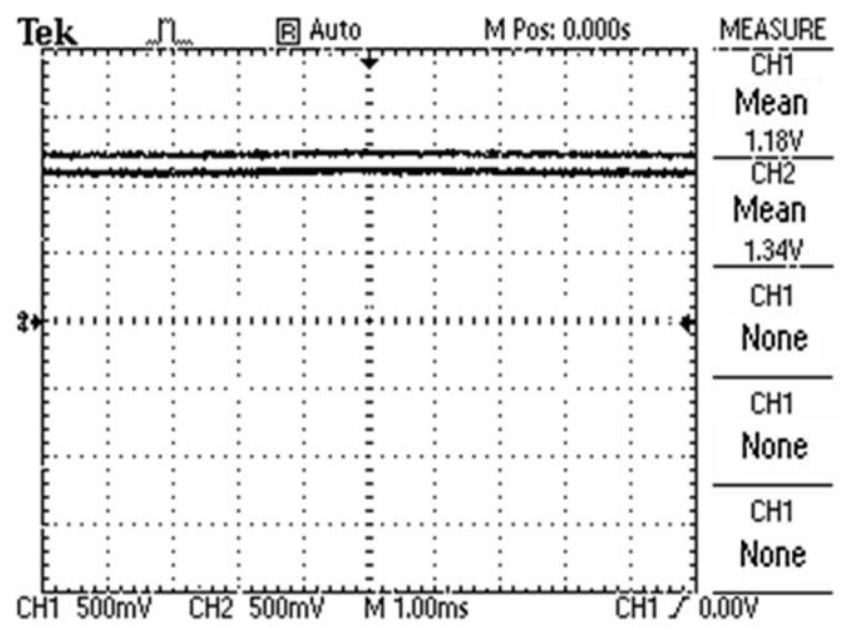

Fig.10. The testing result for the heading angle is -90degrees.

Table 2 gives testing results $V_{L}, V_{R}$, and $V_{\text {ref }}$ for different values of the heading angle. It gives an expected result that the value $V_{r e f}$ is independent of the heading angle. Fig.11 shows measured datum $V_{R}$ and $V_{L}$ for heading errors varying from 90degrees to +90degrees using datum given in Table 2. Fig.11 shows the maximal output of the right loop or left loop is not at the zero heading error (i.e., along the $\mathrm{X}$-axis). They are disturbed by the coupling effect. The disturbance is dependent on the spacing D between two loop antennas.

Fig.12 shows the transfer curve for $V_{R}-V_{L}$ versus the heading angle. It gives a linear relationship between $V_{R}-V_{L}$ and the heading angle. In general, this is the expected property for sensors. The dash lines show expected results over 90degree or less than -90 degrees. It implies that it is repeated from 90degrees to 180degrees; or from -90degrees to 180degrees.
Table 2. Testing results for Range $=6 \mathrm{M}$.

\begin{tabular}{|c|c|c|c|}
\hline $\begin{array}{c}\text { Heading } \\
\text { (Deg.) }\end{array}$ & $\begin{array}{c}\text { Left Loop } \\
V_{L}(\mathrm{~V})\end{array}$ & $\begin{array}{c}\text { Right Loop } \\
V_{R}(\mathrm{~V})\end{array}$ & $\begin{array}{c}\text { Reference } \\
V_{r e f}(\mathrm{~V})\end{array}$ \\
\hline 90.00 & $1.30 \sim 1.38$ & $1.13 \sim 1.21$ & $0.97 \sim 1.03$ \\
\hline 60.00 & $1.34 \sim 1.41$ & $1.24 \sim 1.32$ & $0.99 \sim 1.02$ \\
\hline 45.00 & $1.39 \sim 1.42$ & $1.27 \sim 1.35$ & $1.00 \sim 1.04$ \\
\hline 30.00 & $1.37 \sim 1.45$ & $1.32 \sim 1.39$ & $1.00 \sim 1.04$ \\
\hline 15.00 & $1.40 \sim 1.42$ & $1.36 \sim 1.44$ & $1.00 \sim 1.04$ \\
\hline 0.00 & $1.40 \sim 1.48$ & $1.39 \sim 1.49$ & $1.01 \sim 1.08$ \\
\hline-15.00 & $1.37 \sim 1.42$ & $1.40 \sim 1.45$ & $1.00 \sim 1.04$ \\
\hline-30.00 & $1.37 \sim 1.40$ & $1.39 \sim 1.46$ & $0.99 \sim 1.06$ \\
\hline-45.00 & $1.34 \sim 1.35$ & $1.39 \sim 1.44$ & $0.99 \sim 1.06$ \\
\hline-60.00 & $1.24 \sim 1.32$ & $1.36 \sim 1.42$ & $0.99 \sim 1.05$ \\
\hline-90.00 & $1.16 \sim 1.21$ & $1.33 \sim 1.38$ & $0.97 \sim 1.04$ \\
\hline
\end{tabular}

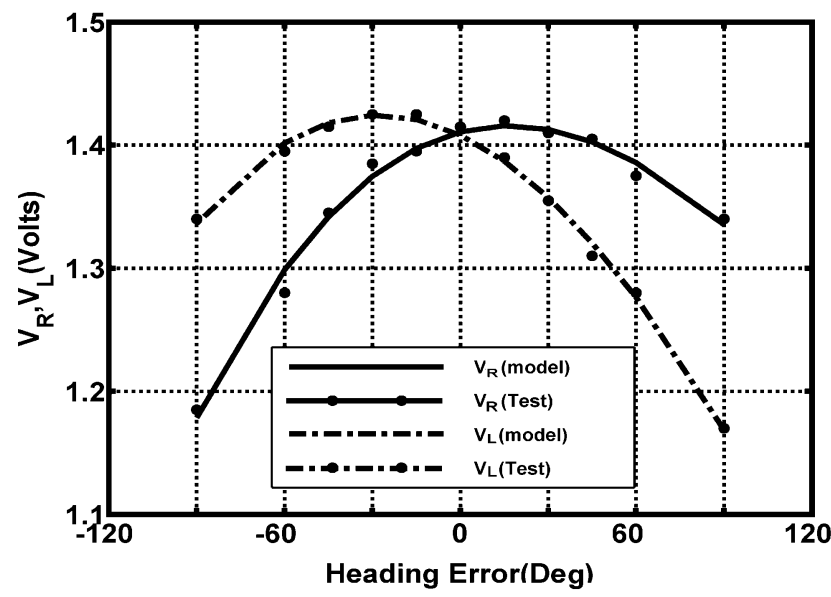

Fig.11. Demodulated Outputs of Right and Left Antennas.

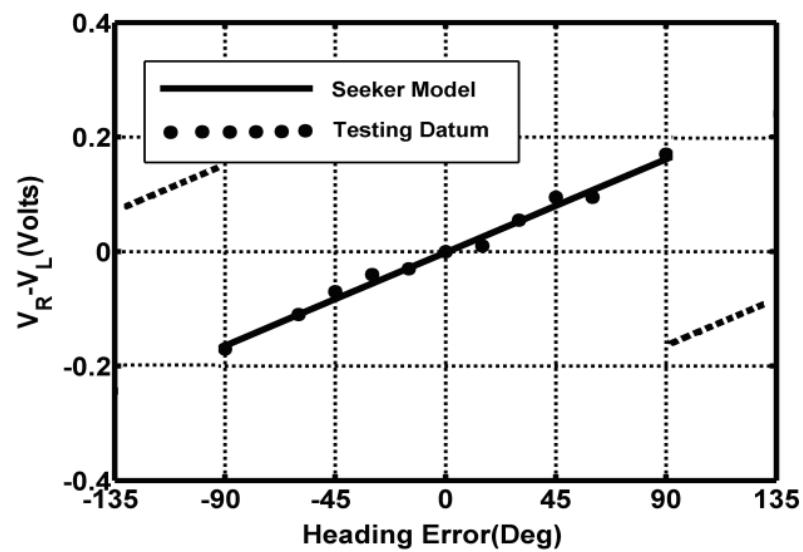

Fig.12. The Transfer curve for $V_{R}-V_{L}$ versus heading error. 
Fig. 12 shows that there are two jumps at -90degrees and +90degrees. Therefore, the operation range is limited from -90degrees to +90degrees. For example, the measurement data for +135 degrees will be equal to that of -45 degrees. This confusion must be prevented for real application. Fig.13 shows a possible implementation using a ground shield on the rear side for preventing this confusion. The difference between $V_{R}$ and $V_{L}$ will become be zero for the heading angle greater than +90degrees or less than -90degree.

The limitation of the proposed loop antenna(shown in Fig.4) can be expended by adding another two loop-antenna in a perpendicular for omnidirectional radio finding on the horizontal plane(X-Y plane).

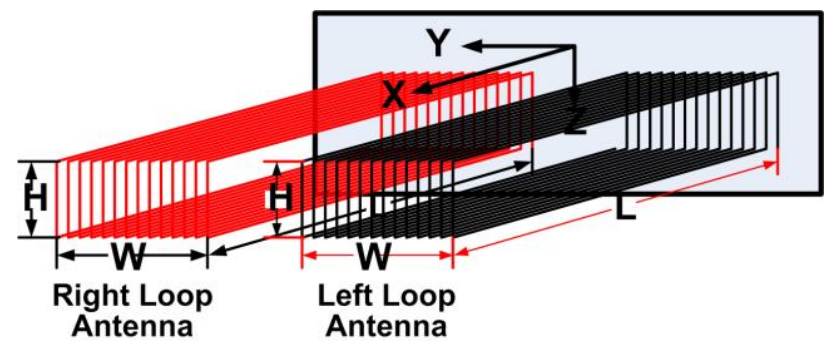

Fig.13. A ground shield for preventing wrong data.

\section{Conclusions}

In this paper, the two coupled-loop antennas and the signal-processing circuit with demodulation have been proposed for radio direction finding. Vast measurements were made and gave a large linear detecting range from -90degree to +90degrees. It is ready for Omni-directional application using another two coupled loop antennas and can be applied to the high-frequency range using a smaller loop antenna.

\section{References:}

[1] H. Whiteside and R. W. P. King, "The loop antenna as a probe", IEEE Transactions on Antennas and Propagation, Vol.12, no. 3, pp. 291-297, 1964.

[2] M. A. Jensen, and Y. Rahmat-Samii, " Electromagnetic characteristics of super-quadric wire loop antennas," IEEE Transactions on Antennas and Propagation, 1994, Vol.42, No.2, pp.264269.

[3] R. L. Li, G. DeJean, J. Laskar, and M. M. Tentzeris, "Investigation of Circularly Polarized Loop Antennas With a Parasitic Element for Bandwidth Enhancement, "IEEE Transactions on Antennas and Propagation, Vol. 53, No. 12, 2005, pp.3930-3939.

[4] A. Boswell, A. J. Tyler, and A. White, " Performance of a small loop antenna in the 3-10 $\mathrm{MHz}$ band," IEEE Antennas and Propagation Magazine, Vol.47, No.22, 2005, pp.51-56.

[5] Y. W. Chi and K. L. Wong," Very-Small-Size Printed Loop Antenna for GSM/DCS/ PCS/UMTS Operation in the mobile phone, "Microwave and Optical Technology Letters, Vol. 51, No. 1, 2009, pp.184-192.

[6] S. Youn, T. H. Lim, E. Kang, D. H. Lee, K. B. Kim and H. Choo, "Design of a Miniaturized Rectangular Multiturn Loop Antenna for Shielding Effectiveness Measurement," Sensors, 2020, 20, 3178; doi:10.3390/s20113178.

[7] T. Tsukiji, "Analysis of two coupled coplanar loops, "IEEE Transactions on Antennas and Propagation, Vol.23, No.2, 1975, pp.250-253.

[8] K. Iizuka, R. King, and C. Harrison, "Self- and mutual admittances of two identical circular loop antennas in a conducting medium and in air, "IEEE Transactions on Antennas and Propagation, 1966, Vol.14, No.4, pp.440-450.

\section{Creative Commons Attribution License 4.0 (Attribution 4.0 International , CC BY 4.0)}

This article is published under the terms of the Creative Commons Attribution License 4.0 https://creativecommons.org/licenses/by/4.0/deed.en US 